\title{
Aprendizaje cooperativo en Educación Física: estado dela cuestión y propuesta de intervención Cooperative learning in Physical Education: the state of the question and intervention proposal Carlos Velázquez Callado \\ Universidad de Valladolid(España)
}

Resumen. En los últimos años varias investigaciones han subrayado las ventajas del aprendizaje cooperativo para promover el aprendizaje motor y social en Educación Física. Sin embargo, rara vez se describe lo que los docentes hacen cuando aplican este modelo pedagógico en sus clases de Educación Física. Este artículo presenta un estudio exploratorio orientado a dar respuesta a esta pregunta. Ciento noventa y ocho profesores de Educación Física españoles respondieron a un cuestionario dirigido a conocer cómo conciben el aprendizaje cooperativo y el modo en que lo implementan en sus clases con alumnado de Primaria y Secundaria. Los resultados del estudio muestran que el aprendizaje cooperativo es todavía una metodología poco implementada en Educación Física. Los profesores la conciben desde una perspectiva cercana al juego cooperativo. Se propone entonces un nuevo enfoque pedagógico, denominado coopedagogía, orientado a facilitar que los docentes puedan desarrollar un proceso de intervención que permita, por una parte, que el alumnado aprenda a cooperar y, por otra, que utilice la cooperación como recurso para aprender en las clases de Educación Física. Palabras clave. Aprendizaje cooperativo, educación física, socialización, aprendizaje activo, práctica educativa.

Abstract. Over the last few years several studies have emphasized the advantages of cooperative learning to promote motor and social learning in Physical Education. However, what teachers do when they implement this pedagogical model in their Physical Education classes is rarely described. This paper presents an exploratory study aimed at answering this question. One hundred and ninety-eight Spanish Physical Education teachers completed a questionnaire designed to find out how cooperative learning is conceived and applied practically in Primary and Secondary school classes. The results of the study reveal that cooperative learning is still underutilized in Physical Education. Teachers conceive cooperative learning from a perspective close to cooperative play. A new pedagogical approach, called coopedagogy, is then proposed to facilitate teachers in developing an intervention process that allows students to learn how to cooperate and how to use the cooperation as a resource of learning in Physical Education classes.

Keywords. Cooperative learning, physical education, socialization, active learning, educational practice.

\section{Introducción}

El aprendizaje cooperativo es una metodología educativa basada en el trabajo en pequeños grupos, normalmente heterogéneos, en los que los estudiantes aúnan esfuerzos y comparten recursos para mejorar su propio aprendizaje y también el de los demás miembros del equipo (Johnson, Johnson \& Holubec, 1999; Velázquez, 2010). La clave de esta definición, lo que diferencia el aprendizaje cooperativo del mero trabajo grupal, es precisamente esa corresponsabilidad en el aprendizaje. Cada estudiante no busca solo aprender un determinado contenido, sino que facilita la ayuda necesaria para que el resto de sus compañeros lo logre también (Dyson, 2002).

Johnson y Johnson (1999) identifican cinco condiciones cuya presencia sería determinante para promover el aprendizaje cooperativo y, en consecuencia, el éxito en el trabajo grupal: (1) interdependencia positiva de metas, pero también de recursos, de roles...; (2) interacción promotora, o manifestación de conductas de ayuda, apoyo y ánimo dentro del grupo; (3) responsabilidad individual, de modo que nadie se escuda en el trabajo de los otros; (4) habilidades interpersonales y de trabajo en pequeño grupo, y (5) procesamiento o autoevaluación grupal, orientada a que los propios estudiantes sean capaces de identificar las conductas manifestadas durante el trabajo grupal, relacionándolas, positiva o negativamente, con los logros alcanzados.

El aprendizaje cooperativo no solo favorece el logro de objetivos académicos en distintas áreas y niveles educativos (Byra, 2006; Johnson, Johnson \& Stanne, 2000), sino que además promueve objetivos del ámbito afectivo y social (Gillies \& Boyle, 2010; Pujolàs, 2008). Más específicamente, encontramos investigaciones que demuestran la eficacia de esta metodología para favorecer unas relaciones interpersonales positivas entre el alumnado, lo que le convierte en un excelente recurso metodológico para fomentar un clima positivo de clase(Ghaith, Shaaban \& Harkous, 2007) que promueva la inclusión del alumnado con discapacidad (Piercy, Wilton \& Townsend, 2002), la coeducación (Petersen, Johnson \& Johnson, 1991) o la inclusión de minorías étnicas (Díaz Aguado \& Baraja, 1993; Oortwijn, Boekaerts, Vedder \& Fortuin, 2008). Son también frecuentes los estudios que asocian el aprendizaje cooperativo con un aumento de la motivación del alumnado hacia elárea

Fecha recepción: 09-12-14- Fecha envío revisores: 09-12-14- Fecha de aceptación: 02-02-15

Carlos Velázquez Callado

cvelazquez@mpc.uva.es de estudio (Hänze \& Berger, 2007; Marín \& Blázquez, 2003; Tarim \& Akdeniz, 2008) y con una mejora del autoconcepto de los estudiantes (Denigri, Opazo \& Martínez, 2007; Johnson, Johnson \& Taylor, 1993; Kirk, 1999).

Con todo ello, podemos concluir que el aprendizaje cooperativo se presenta actualmente como uno de los recursos metodológicos más eficaces a la hora de promover el logro social y académico del alumnado en diferentes áreas, niveles y contextos de aprendizaje.

El aprendizaje cooperativo en Educación Física: análisis de la literatura

La aplicación del aprendizaje cooperativo en el ámbito motor es algo relativamente reciente. Así, aun cuando podemos encontrar en la literatura algunos estudios previos al siglo XXI (Stanne, Johnson \& Johnson, 1999; Strachan, 1996), la mayor parte de las investigaciones referidas a la aplicación de esta metodología en Educación Física y deporte son del presente siglo.

Velázquez (2012) señala que la introducción del aprendizaje cooperativo en Educación Física tiene como punto de partida la Teoría de la cooperación y la competición (Deutsch, 1949) y su aplicación en dos grandes ámbitos. Por una parte, en la regulación de los conflictos y la educación para la paz y, por otra, en contextos de educación formal. En el primer caso, la búsqueda de un modo de jugar basado en una interdependencia positiva de metas generó trabajos prácticos de creación e implementación de programas de juegos cooperativos (Provost \& Villeneuve, 1980; Orlick, 1978). En el segundo, la aplicación de la teoría de Deutsch (1949) en el ámbito educativo derivó en la Teoría de la interdependencia social (Johnson \& Johnson, 1989) y en el aprendizaje cooperativo propiamente dicho.

La investigación sobre las posibilidades del juego motor cooperativo en contextos educativos destaca que puede ser un excelente recurso de inclusión (Grineski, 1989; Marín, 2007), que además promueve conductas prosociales entre el alumnado (Garaigordobil, 2005; Street, Hoppe, Kingsbury \& Ma, 2004) al tiempo que disminuye los comportamientos problemáticos en las clases (Bay-Hinitz, Peterson \& Quilitch, 1994; Garaigordobil, 2004). Otros logros atribuidos a programas de juegos cooperativos son mejorar el autoconcepto del alumnado (Garaigordobil, 2003, 2004), promover la creatividad (Garaigordobil, 2004, 2005, 2007) o aumentar la motivación hacia la práctica motriz (Marín, 2007).

Ahora bien, juego cooperativo y aprendizaje cooperativo no pueden ser considerados términos sinónimos. Velázquez (2004) define el 
juego cooperativo como una actividad lúdica colectiva en la cual no existe oposición entre las acciones de los participantes, sino que, por el contrario, todos aúnan esfuerzos para alcanzar un mismo fin o varios objetivos complementarios. Desde esta definición encontramos algunas similitudes con el aprendizaje cooperativo. La principal es que existe una interdependencia positiva de metas. También es muy probable que, durante la práctica motriz, se manifiesten conductas mutuas deánimo y de ayuda entre los jugadores. Además, estos pondrán en práctica sus habilidades sociales a la hora de afrontar los problemas que el juego les plantea y de alcanzar acuerdos que les permitan resolverlos. Sin embargo, Velázquez (2012) plantea algunas diferencias entre el juego y el aprendizaje cooperativo. La primera es que el juego cooperativo es una actividad con un carácter puntual, mientras que el aprendizaje cooperativo es una metodología y, por lo tanto, una práctica pedagógica extendida en el tiempo. La segunda diferencia hace referencia a la finalidad que pretenden. El juego cooperativo busca la diversión de los participantes, aun cuando el docente pueda utilizarlo para promover objetivos didácticos. El aprendizaje cooperativo pretende que el alumnado aprenda, con independencia de que el proceso sea más o menos divertido. Una tercera diferencia tiene que ver con la evaluación. Mientras que la autoevaluación grupal es uno de los componentes esenciales del aprendizaje cooperativo, en el juego cooperativo solo es aconsejable. Un cuarto elemento que los diferencia está relacionado con la responsabilidad del alumnado. Mientras que en el aprendizaje cooperativo la responsabilidad individual es ineludible, hasta el punto de no es posible que el grupo alcance su objetivo si alguno de sus componentes se escuda en el trabajo de los demás, en el juego cooperativo solo es necesaria la responsabilidad grupal. De hecho, un jugador puede adoptar libremente una actitud pasiva durante la práctica motriz sin que ello repercuta negativamente en el logro colectivo. Finalmente, la propia definición de juego cooperativo hace imposible la existencia de oposición entre las acciones de los participantes; sin embargo, algunas técnicas de aprendizaje cooperativo, como el Torneo de Juegos por Equipos (DeVries \& Edwards, 1972), incluyen la competición intergrupal durante su implementación (tabla1).

Tabla 1.

Diferencias entre eljuego cooperativo y el aprendizaje cooperativo (Velázquez, 2012, p.88).

\begin{tabular}{ll} 
Diferencias entre eljuego cooperativo yel aprendizaje cooperativo (Veläzquez, 2012,p.88). \\
\hline Juego cooperativo Aprendizaje cooperativo
\end{tabular} \begin{tabular}{ll} 
Juego cooperativo & Aprendizaje cooperativo \\
\hline Actividad puntual & Metodología. Práctica pedagógica exten dida en el tiempo
\end{tabular} \begin{tabular}{ll} 
Actividad puntual & Metodología. Práctica pedagógica extendida en el tiempo \\
\hline Principal objetivo: diversión & Principal objetivo: aprendizaje \\
\hline
\end{tabular}

Evaluaciónopciana $\begin{array}{ll}\text { No cinco componentes, } & \text { interacción promotora, respon sabilidad individual, }\end{array}$ $\begin{array}{ll}\text { los cinco componentes, } & \text { interacción promotora, resp on sabilidad individual, } \\ \text { especialmente la responsabilidad } & \text { habilidades interpersonales y de pequeño grupo y }\end{array}$ individual (solo grupal) procesamiento grupal

No existe oposición entre las Puede presentar competición intergrupa acciones de los participantes

Así, podemos afirmar que, aun cuando el juego cooperativo puede suponer un primer paso hacia el aprendizaje cooperativo, el docente debe seguir avanzando para promover en sus clases la presencia de los diferentes factores que favorecen el aprendizaje desde el trabajo grupal.

Como ya hemos mencionado, la aplicación del aprendizaje cooperativo en las clases de Educación Física es relativamente reciente. En 1996, Grineski realizó un estudio con 500 estudiantes de las etapas de educación obligatoria, concluyendo que más del $90 \%$ de sus experiencias en Educación Física habían sido de tipo competitivo. De hecho, las primeras investigaciones orientadas a evaluar la eficacia del aprendizaje cooperativo se centraron en áreas con un alto componente cognitivo, como pueden ser Lenguaje, Ciencias o Matemáticas (DeVries \& Slavin, 1976; Johnson, Maruyama, Johnson, Nelson \& Skon, 1981) y solo a principios del presente siglo comienzan a publicarse algunos estudios realizados en Educación Física.

La mayor parte de las investigaciones intentan determinar los efectos derivados de la aplicación del aprendizaje cooperativo en Educación Física, tanto sobre el aprendizaje motor como sobre el desarrollo de objetivos sociales y afectivo-motivacionales. Aunque resulta complicado comparar estudios para extraer unas conclusiones generales (debido a las diferencias contextuales existentes entre ellos, centradas en el nivel y etapa educativa, el enfoque o técnicas de aprendizaje cooperativo empleados, el contenido trabajado o la duración de la investigación) todo parece indicar que el aprendizaje cooperativo, correctamente implementado, se muestra superior a las metodologías tradicionales a la hora de favorecer en el alumnado el desarrollo de conductas prosociales (Dyson, 2001, 2002; Goudas \& Magotsiou, 2009; Polvi \& Telama, 2000; Prieto \& Nistal, 2009), la responsabilidad individual (Dyson, Linehan \& Hastie, 2010) y objetivos afectivo-motivacionales (Barba, 2010; Fernández-Río, 2003). Otras investigaciones destacan el papel del aprendizaje cooperativo para fomentar la inclusión del alumnado con discapacidad en las clases de Educación Física (André, Deneuve \& Louvet, 2011; Dowler, 2012; Velázquez, 2012b).

Con respecto al aprendizaje motor, la superioridad del aprendizaje cooperativo en relación a otras metodologías más tradicionales no está tan clara. Así, existen estudios comparativos que concluyen subrayando la efectividad del aprendizaje cooperativo sobre métodos directivos a la hora de promover el aprendizaje motor en Educación Física (André, 2012; Bayraktar, 2011; Barrett, 2005), mientras que otros señalan la inexistencia de diferencias significativas entre ambas opciones metodológicas (Gröben, 2005; Prieto \& Nistal, 2009). Otros estudios no comparativos resaltan que, tras un necesario período de adaptación del docente y del alumnado a las exigencias de la nueva metodología, el tiempo de práctica motriz aumenta y se alcanzan los objetivos de aprendizaje dentro de los niveles previstos si se utilizasen otras metodologías más tradicionales (Barba, 2010; Dyson, 2001, 2002; Dyson, Linehan \& Hastie, 2010; Velázquez, 2012b). Finalmente, algunas investigaciones subrayan la importancia de algunos factores para promover el logro motor cuando se trabaja con el aprendizaje cooperativo, como las interacciones verbales (Lafont, Proeres \& Vallet, 2007) o la responsabilidad y la implicación activa de los estudiantes en la tarea encomendada (Bähr, 2010; Casey \& Dyson, 2009).

Todo ello parece indicarnos que la introducción del aprendizaje cooperativo en Educación Física beneficiaría la consecución de objetivos sociales y afectivo-motivacionales del alumnado sin que el logro motor disminuyera. Además, esta metodología se convierte en un recurso de referencia para promover la inclusión del alumnado que presenta algún tipo de discapacidad en las clases de Educación Física.

\section{La práctica del aprendizaje cooperativo en Educación Física. Un} estudio exploratorio

La investigación presenta al aprendizaje cooperativo como un potente recurso metodológico para promover tanto el aprendizaje motor como el desarrollo de conductas prosociales y la inclusión de todo el alumnado. La pregunta es hasta qué punto y cómo lo aplican los docentes deEducación Física en sus clases. En este sentido, se planteó realizar un estudio aproximativo, con docentes españoles, que abordara esta cuestión.

\section{Metodología}

\section{Recogida de datos}

Se elaboró un cuestionario exploratorio compuesto por un conjunto de preguntas, generalmente cerradas de opción múltiple o semiabiertas, orientadas a recabar información sobre las características de los docentes entrevistados y del contexto en el que trabajaban, sobre su conocimiento del aprendizaje cooperativo y, en el caso de que lo aplicasen en sus clases, sobre el modo en que lo implementaban con su alumnado.

Una primera versión del cuestionario fue sometida a un proceso de validación por expertos, realizándose las modificaciones necesarias hasta su aprobación. A continuación se aplicó a un pequeño grupo de 10 docentes de Educación Física de distintas comunidades autónomas, para subsanar posibles problemas de comprensión o de ambigüedad en alguna de las preguntas. La versión definitiva del cuestionario se remitió a todos los centros españoles de formación permanente del profesorado, a las principales asociaciones de docentes de Educación Física y a los colegios profesionales de licenciados en Educación Física. En todos los casos se pidió ayuda para su distribución entre el profesorado de sus respectivos ámbitos. 


\section{Participantes}

Se recibieron las respuestas de 198 docentes españoles que impartían docencia directa de Educación Física con alumnado de Educación Primaria o Secundaria Obligatoria.

\section{Análisis de los datos}

Para el análisis de los datos se consideraron dos grandes núcleos temáticos: (1) conocimiento y concepción del aprendizaje cooperativo, y (2) aplicación de esta metodología con alumnado. Se realizó un análisis de frecuencias en las preguntas de opción múltiple y se procedió a un análisis de contenido en las preguntas semiabiertas, apoyándonos en el programa «Atlas.ti.6.2». En este sentido, para el segundo de los núcleos temáticos se consideraron diferentes categorías, entre las que destacamos: objetivos desarrollados, contenidos trabajados con aprendizaje cooperativo, técnicas utilizadas, agrupamientos, materiales proporcionados al alumnado y proceso de evaluación.

\section{Resultados}

El primer dato relevante es que un $22.2 \%$ de los docentes que contestaron al cuestionario manifestaron no conocer el aprendizaje cooperativo. El análisis de las respuestas del otro $77.8 \%$ restante ( $\mathrm{n}=$ 154) nos permitió comprobar que la gran mayoría de los docentes que afirman conocer el aprendizaje cooperativo lo entienden como un término genérico en el que se integran diferentes procesos de aprendizaje grupal, que incluyen desde el trabajo en grupo hasta el juego cooperativo. Así, un docente lo entiende como «el aprendizaje a través del trabajo e intercambio de experiencias con compañeros, colaborando en la realización de actividades y la búsqueda de soluciones» mientras que para otro consiste en «juegos sin eliminados, deportes y juegos no competitivos basados en el trabajo grupal y la búsqueda común de un objetivo». Algunos profesores, identifican el aprendizaje cooperativo con un proceso de tutoría entre iguales, mediante el cual cuando «alguno de los compañeros domina medianamente el contenido, ayuda a progresar a sus compañeros». A pesar de esta diversidad de puntos de vista, la mayor parte del profesorado concibe el aprendizaje cooperativo como un proceso en grupo que «pretende no solo la actuación conjunta de los alumnos en pos de un fin común, sino el trabajo aunado de todos para conseguir dicho objetivo». En definitiva, implica objetivos comunes y una necesaria coordinación de esfuerzos entre el alumnado.

De los 154 docentes que afirmaban conocer el aprendizaje cooperativo, el 15.4\% reconoce no utilizarlo en sus clases, de forma que el análisis del modo en que esta metodología es aplicada en Educación Física se basa en las respuestas del $84.6 \%$ restante $(n=130)$. Profundizando un poco más, este grupo estaba compuesto por 52 mujeres y 77 hombres (una persona no manifestó su sexo), de edades comprendidas entre los 25 y los 55 años $(M=35.98, \sigma=6.84)$ y con una experiencia impartiendo clase de Educación Física de entre uno y 35 años $(M=$ 10.37, $\sigma=6.47$ ). La mayor parte de los docentes trabajaban en escuelas públicas (88.5\%). Por etapas educativas, el 68.5\% impartía Educación Física en Primaria, el $26.9 \%$ en Secundaria y un $4.6 \%$ lo hacía en ambas.

El profesorado manifiesta utilizar el aprendizaje cooperativo en sus clases para alcanzar no solo objetivos motores, sino también, y muy especialmente, sociales y afectivo-motivacionales. Una profesora de Educación Primaria resume esta idea señalando que «no solo se trabaja el nivel motor y conceptual, sino también el emocional y actitudinal».

Los contenidos trabajados mediante aprendizaje cooperativo en Educación Física son múltiples y variados, de modo que algunos docentes lo emplean en unidades didácticas en las que se desarrollan propuestas sociomotrices, como el acrosport o actividades colectivas de expresión corporal, mientras que otros lo incorporan en actividades con una estructura manifiestamente competitiva, como los deportes de equipo.

Es poco habitual que los docentes utilicen técnicas estructuradas de aprendizaje cooperativo, de hecho solo el 36.2\% manifiesta hacerlo en sus clases. La mayor parte de las respuestas hacen referencia únicamente a juegos cooperativos o a trabajo en grupo en el que se resaltan una serie de conductas que el profesorado quiere destacar al alumnado. Un profesor de Educación Secundaria afirma que «en todos los contenidos que trabajamos se trata de resaltar ante todo el valor del trabajo en equipo, la ayuda y colaboración con el resto de compañeros y compañeras... por encima de resultados». Entre los pocos docentes que sí plantean técnicas específicas de aprendizaje cooperativo, las más frecuentemente mencionadas son, en este orden: (1) piensa - comparte actúa (Grineski, 1996), por norma general orientada al desarrollo de desafíos físicos cooperativos, (2) el rompecabezas o puzle (Aronson, Blaney, Stepahn, Sikes \& Snapp, 1978), (3) la enseñanza recíproca (Mosston, 1978), y (4) el marcador colectivo (Orlick, 1990).

A la hora de agrupar al alumnado, la mayoría de los docentes (86.2 \%) están de acuerdo en considerar importante que los grupos de aprendizaje cooperativo sean heterogéneos. Entre los criterios de heterogeneidad que el profesorado tiene en cuenta, el principal es el nivel de habilidad motriz con respecto a la tarea que va a ser trabajada, seguido del sexo y del grupo étnico. Los grupos suelen estar formados por cuatro o cinco estudiantes y la tendencia del profesorado es mantener los grupos estables poco tiempo. Así, el $58.5 \%$ de los docentes manifiesta que la duración de los grupos es de una sesión de clase o menos. Solo un 16.2 $\%$ afirma mantener los grupos durante una unidad didáctica y un 7.7\% indica que cambia de grupos cada trimestre.

Aunque un poco más de la mitad de los docentes (54.6\%) afirma que elaboran materiales didácticos específicamente diseñados para trabajar con aprendizaje cooperativo, el análisis cualitativo de sus respuestas nos muestra que la gran mayoría se refieren a unidades didácticas o actividades concretas de estructura cooperativa y no tanto a materiales que sirven al alumnado de apoyo cuando trabaja con esta metodología. Así, un profesor de Educación Secundaria explica que él diseña «juegos modificados para trabajar la educación en valores a través de estrategias cooperativas», mientras que otro de Primaria señala que plantea «juegos de aventura, cuentos motrices y algunas unidades didácticas ambientadas». Centrándonos solo en materiales orientados a facilitar el aprendizaje autónomo del alumnado mediante aprendizaje cooperativo, los más frecuentemente mencionados por el profesorado hacen referencia a recursos orientados a la autoevaluación y coevaluación de los aprendizajes, como «hojas de control de su aprendizaje en salto de comba, instrumentos de evaluación del juego (GPAI), fichas de registro de datos en carrera de larga duración...», o «cuestionarios de evaluación en cada unidad didáctica». También son relativamente frecuentes las fichas orientadas a explicar la tarea que el alumnado va a realizar en los grupos «con claves del contenido a trabajar y seguimiento del aprendizaje».

A pesar de que el procesamiento grupal es considerado un componente esencial del éxito del aprendizaje cooperativo, únicamente el 35.4\% de los docentes encuestados manifiesta destinar un tiempo a que cada grupo reflexione, de forma independiente del resto, sobre el trabajo realizado y las conductas que se han manifestado durante el mismo. Sin embargo, el 54.6\% de los docentes afirma promover esa reflexión de forma colectiva, en gran grupo.

A la hora de calificar a su alumnado, el 56.2\% del profesorado encuestado que implementa el aprendizaje cooperativo en sus clases tiende a valorar tanto el aprendizaje motor como diferentes objetivos sociales y actitudinales. Un $5.4 \%$ califica solo con base en el grado de logro motor y social del alumnado y un $6.2 \%$ lo hace considerando exclusivamente objetivos motores y actitudinales. Casi un tercio de los docentes que respondieron al cuestionario (30.8\%) no concede importancia a los resultados motores, manifestando que únicamente califica los aspectos sociales y/o los actitudinales cuando trabaja con aprendizaje cooperativo. En el otro extremo, solo un docente afirmó calificar considerando únicamente el logro motor de su alumnado.

\section{Discusión}

En los últimos años el aprendizaje cooperativo se está convirtiendo en una metodología básica dentro del modelo de educación basado en competencias (Blázquez \& Bofill, 2009; Johnson \& Johnson, 2014; Velázquez, 2012). Sin embargo, los resultados de nuestro estudio mues- 
tran que ni es conocida por todos los docentes de Educación Física, ni todos la entienden de la misma manera. Así, la delimitación entre lo que es aprendizaje cooperativo, trabajo en grupo y juego cooperativo no está tan clara entre el profesorado, de modo que los docentes con frecuencia mezclan características específicas de cada uno de estos conceptos e identifican unos con otros.

El profesorado que manifiesta utilizar el aprendizaje cooperativo en sus clases tiene claro que estas deben favorecer el logro motor de su alumnado, si bien entiende que esta metodología promueve, además de ello, el desarrollo de objetivos de carácter social y afectivo-motivacional, que también considera fundamentales. Otros autores coinciden en el hecho de que los docentes que optan por implementar el aprendizaje cooperativo en sus clases de Educación Física lo hacen buscando no solo el logro motor, sino el desarrollo de metas sociales y afectivas (Casey, Dyson \& Campbell, 2009; Dyson, 2001, 2002; Dyson, Linehan \& Hastie, 2010; Fernández-Río, 2003). El hecho de que la mayor parte de los docentes califique a su alumnado valorando tanto el logro motor como objetivos sociales y actitudinales, apunta también en este sentido.

La diversidad de contenidos trabajados mediante aprendizaje cooperativo afianza la idea de que cualquier contenido puede ser enseñado mediante esta metodología (Johnson \& Johnson, 1999; Velázquez, Fraile \& López-Pastor, 2014). El hecho de que un buen número de docentes manifieste utilizar el aprendizaje cooperativo para la enseñanza deportiva muestra que entienden que la forma de estructurar el proceso de aprendizaje es independiente de la estructura de la actividad que debe ser enseñada (Velázquez, 2010, 2012).

Los docentes participantes en nuestro estudio no tienden a utilizar técnicas estructuradas de aprendizaje cooperativo en Educación Física, a pesar de que un buen número de publicaciones las describen detalladamente(Grineski, 1996; Velázquez, 2004, 2010, 2012). Por el contrario, plantean el trabajo en grupo incidiendo en el alumnado en la necesidad de coordinar esfuerzos y responsabilidades para alcanzar un objetivo común, algo que, como afirman diferentes autores, no se puede considerar aprendizaje cooperativo (Cohen, 1999; Johnson \& Johnson, 1999; Kagan, 2000). Los pocos docentes que manifiestan implementar algunas técnicas de aprendizaje cooperativo se decantan, en la mayoría de los casos por aquellas cercanas al juego cooperativo, como «piensacomparte-actúa» (Grineski, 1996) o «marcador colectivo» (Orlick, 1990).

La práctica totalidad del profesorado entiende que es positivo el trabajo en grupos heterogéneos, algo en lo que también coinciden los principales referentes del aprendizaje cooperativo (Johnson \& Johnson, 1999; Kagan, 2000; Pujolàs, 2008; Slavin, 1999). Sin embargo, al contrario de lo que se aconseja en la literatura, solo un reducido porcentaje de docentes apuesta por una cierta estabilidad de los grupos en las clases de Educación Física. Esto nos lleva a pensar que, en muchos casos, el profesorado no se está refiriendo a aprendizaje cooperativo, sino a actividades cooperativas o a trabajo grupal. El hecho de que sean pocos los profesores que utilicen materiales didácticos específicamente diseñados para facilitar que el alumnado pueda aprender de forma autónoma y de que el procesamiento grupal no se realice en los propios grupos, sino con toda la clase, de forma general, refuerzan aún más esta afirmación.

\section{Conclusiones}

Nuestro objetivo era el de hacer un estudio exploratorio orientado a comprobar hasta qué punto y de qué manera aplican los docentes de Educación Física españoles el aprendizaje cooperativo en sus clases. Insistimos en la idea de que nunca ha sido nuestra intención generalizar resultados y obviamente el número de docentes que respondieron a nuestro cuestionario tampoco lo permite, por lo que nuestras conclusiones deben ser tomadas con la debida cautela.

En principio, debemos destacar que el profesorado que dice aplicar el aprendizaje cooperativo en sus clases parte de un planteamiento de educación integral de su alumnado. Así, considera tan importante el desarrollo de la competencia motriz como promover un ambiente positivo en las clases de Educación Física, que facilite la inclusión y la participación activa de todos sus estudiantes. De este modo, las metas sociales y afectivo-motivacionales se sitúan al mismo nivel que el logro motor.

Para el desarrollo de los contenidos curriculares, desde esa visión de educación integral de sus educandos, la mayoría de los docentes introducen diferentes recursos en las clases de Educación Física entre los que destacan el trabajo grupal y el juego cooperativo. Este proceso es considerado por muchos docentes como aprendizaje cooperativo. Dicho en otras palabras, identifican las tareas realizadas en las clases de forma grupal o las actividades físicas cooperativas con el aprendizaje cooperativo, lo cual quizás sea una de las causas por las que no profundizan más en esta metodología.

Algunos docentes van un poco más lejos e introducen, de forma puntual, algunas propuestas mediante técnicas estructuradas de aprendizaje cooperativo de corta duración, para el desarrollo de contenidos concretos.

Son muy pocos los profesores que avanzan más allá, planteando procesos de aprendizaje cooperativo prolongados en el tiempo, al menos durante una unidad didáctica, con técnicas en las que el alumnado asuma una amplia autonomía a la hora de organizar, desarrollar y evaluar su propio aprendizaje, de modo que pueda tomar decisiones que extrapolar a otras situaciones.

\section{Física}

Coopedagogía: la pedagogía de la cooperación en Educación

Desde nuestro punto de vista, el proceso de aproximación del profesorado al aprendizaje cooperativo puede incluir los juegos cooperativos, la resolución de problemas en grupo o el refuerzo de las condiciones deseables para generar un ambiente social cooperativo en las clases de Educación Física. Ahora bien, hemos de tener en cuenta que son pasos que recorrer, pero no el final del camino. No es que los docentes estén en un camino equivocado, es solo que necesitan avanzar más. En este sentido, proponemos la introducción de un término más amplio, el de coopedagogía, que se define como el «enfoque educativo orientado a promover que el alumnado aprenda a cooperar y utilice las posibilidades que la cooperación le ofrece para alcanzar eficazmente diferentes aprendizajes curriculares» (Velázquez, 2014, p.45).

El enfoque de coopedagogía, o pedagogía de la cooperación, se plantea con un desarrollo en cinco fases: (1) provocar conflicto, (2) desarrollar los principios de la lógica de la cooperación, (3) aplicar la lógica de la cooperación en ambientes lúdicos, (4) aprender a través de la cooperación, y (5) generar aprendizaje autónomo.

En grupos poco o nada habituados a cooperar o con personas excesivamente individualistas o competitivas, parece coherente que el primer paso se oriente a poner en duda las ideas previas del alumnado. En otras palabras, vamos a cuestionar que competir con los otros o trabajar individualmente genere más beneficios que cooperar. Y vamosa hacerlo aún teniendo en cuenta que «muchas de las condiciones escolares conducen a los estudiantes a creer que su objetivo debe ser superar a los demás o que lo que uno sabe no debe ser compartido con los demás» (Velázquez, 2012, p.135). Para ello, podemos apoyarnos en actividades motrices de estructura compartida (Velázquez, 2004).

Una vez que el alumnado llega al convencimiento de que aunar esfuerzos con los compañeros y compañeras de clase es más efectivo que tratar de ser mejor que ellos o trabajar individualmente puede entender que aprender a cooperar es beneficioso, aunque no sencillo. Nuestro siguiente paso se orienta entonces a que el alumnado entienda la lógica de la cooperación, que tiene como principios básicos que nadie puede sentirse mal en las clases y que todos debemos preocuparnos por todos. Ello nos lleva, por una parte, a identificar en las clases situaciones contrarias a los fundamentos de un ambiente cooperativo y, por otra, a consensuar con el alumnado un conjunto de reglas razonadas que promuevan un proceso de transformación entre el clima de clase que nos encontramos y el que nos gustaría encontrarnos. En definitiva, la cooperación se concreta en un contexto, fundamentado en la responsabilidad y no en la obediencia, que parte de un consenso de normas entre estudiantes y docente. 
Desde esos principios es posible plantear propuestas motrices de resolución cooperativa de problemas o actividades y juegos cooperativos. Se trata entonces de aplicar la lógica de la cooperación en ambientes lúdicos, de divertirse cooperando, de superar retos que implican cada vez más habilidades sociales y destrezas de trabajo en equipo. La introducción de juegos cooperativos sin haber generado previamente una lógica de la cooperación puede desencadenar la manifestación de comportamientos inadecuados como actuar individualmente, perjudicando incluso las respuestas cooperativas de otros compañeros, o buscar la competición, comparando los resultados obtenidos en un grupo con los de otros grupos (Lavega, Planas \& Ruiz, 2014). Estas conductas no hacen sino reforzar la idea de que los estudiantes necesitan aprender a cooperar.

El siguiente paso implica aplicar las habilidades de trabajo en equipo, que se han ido desarrollando en las fases anteriores a facilitar el aprendizaje de contenidos propios del área de Educación Física. Y ello nos lleva al aprendizaje cooperativo. El docente puede comenzar introduciendo técnicas muy estructuradas, con unos pasos claramente establecidos, que garanticen la interacción de los estudiantes y eviten una participación desigual en los grupos. «Marcador colectivo» (Orlick, 1990), «Tres vidas» (Velázquez, 2012), «Yo hago, nosotros hacemos» o «Descubrimiento compartido (Velázquez, 2003) son algunos ejemplos de posibles estructuras específicamente diseñadas para ser aplicadas en Educación Física. Se puede entonces avanzar introduciendo desafíos físicos cooperativos (Glover \& Midura, 1992) u otras propuestas similares que impliquen un proceso de «Piensa-comparte-actúa» (Grineski, 1996). Puede ser el momento de presentar al alumnado las tareas mediante fichas descriptivas del problema que hay que resolver y las condiciones o reglas para hacerlo. El hecho de plantear por escrito tareas de fácil comprensión, en lugar de hacerlo verbalmente, puede facilitar que los estudiantes se vayan habituando a manejar fichas, primero con poca información, que más adelante pueden convertirse en pequeños dosieres que incluyan las claves del proceso de aprendizaje y los instrumentos de evaluación para valorar sus logros. Los siguientes pasos se orientan a la implementación de técnicas menos estructuradas de aprendizaje cooperativo, más prolongadas en el tiempo y que conllevan un objetivo claramente definido y una mayor implicación de los estudiantes a la hora de tomar decisiones que afectan a su propio aprendizaje. Es fundamental entonces que el docente proporcione a los grupos materiales específicamente diseñados para facilitar su aprendizaje autónomo. En este sentido, técnicas como el «Puzle» (Aronson, et al., 1978), «Enseñanza recíproca» (Mosston 1978) o «Equipos de aprendizaje» (Grineski, 1996) pueden apoyarse en dos tipos de recursos: fichas de tarea, que describen qué es lo que el alumnado tiene que hacer y cuáles son las claves de aprendizaje para lograrlo (Casey, 2010), y materiales para la autoevaluacióny la coevaluación(López-Pastor, Barba, Vacas \& Gonzalo, 2010).

El último paso en el marco de la coopedagogía supone que los estudiantes sean capaces de organizarse y de trabajar en equipo autónomamente para desarrollar un determinado proyecto sin la supervisión del docente. En este sentido, aconsejamos el planteamiento de tareas que impliquen aplicar lo aprendido en Educación Física y conlleven un trabajo fuera de horas de clase. Desde nuestro punto de vista el hecho de desarrollarse fuera del horario lectivo supone que las tareas grupales se planteen como voluntarias y con un cierto nivel de complejidad en forma de reto. De esta forma, los estudiantes que se sientan motivados a ir más allá de lo aprendido en las sesiones de Educación Física pueden organizarse libremente, atendiendo a criterios de afinidad o de compatibilidad horaria. Con el fin de no dejar fuera de los grupos a nadie, es conveniente plantear horquillas numéricas en lugar de definir equipos con un número cerrado de integrantes (Pérez-Pueyo, 2005). Inicialmente puede ser conveniente que en los grupos se establezca el compromiso individual que cada estudiante adquiere con el resto de sus compañeros. Si fuera necesario, puede reflejarse por escrito en forma de contrato. A partir de ese momento son los propios grupos los que deben planificar y desarrollar la tarea, regulando los posibles conflictos que pudieran surgir, hasta alcanzar el resultado final.
El enfoque de coopedagogía permite al docente estructurar y poner en práctica un proceso de intervención que parte de un contexto en el que las experiencias previas del alumnado derivan de una estructuración competitiva o individualista del aprendizaje. Mediantela implementación de diferentes acciones, adaptadas a las características del grupo, con base en los cinco niveles expuestos, es posible conducir progresivamente a dicho grupo hasta la meta que buscamos, que no es otra que los estudiantes sean capaces movilizar las habilidades cooperativas aprendidas para organizarse y trabajar en equipo eficazmente sin la supervisión de un adulto. En definitiva, que se muestren competentes a la hora de cooperar para alcanzar sus propios objetivos en la vida y de ayudar a otras personas a lograr los suyos.

\section{Referencias}

André,A. (2012). Influence del'Apprentissage Coopératif sur le savoirs'échauffer et la motivation autodéterminée vis à vis de l'échauffement. eJRIEPS, 27, 5-26. Recuperadodehttp://www.fcomte.iufm.fr/ejrieps

André, A., Deneuve, P. \& Louvet, B. (2011): Cooperative Learning in Physical Education and acceptance of students with learning disabilities. Journal of applied sport Psychology, 23(4), 474-485.

Aronson, E., Blaney, N., Stephan, C., Sikes, J. \& Snapp, M. (1978). The jigsaw classroom. Beverly Hills, CA: SAGE.

Bähr, I. (2010). Experiencia práctica y resultados empíricos sobre el aprendizaje cooperativo en gimnasia. En C. Velázquez (Coord.), Aprendizaje cooperativo en Educación Física. Fundamentos y aplicaciones prácticas (149-163). Barcelona: INDE.

Barba, J. J. (2010). Diferencias entre el aprendizaje cooperativo y la asignación de tareas en la Escuela Rural. Comparación de dos estudios de caso en una unidad didáctica de acrosport en segundo ciclo deprimaria. Retos: nuevas tendencias en educación física, deportes y recreación, 18, 14-18.

Barrett, T. (2005). Effects of cooperative learning on the performance of sixth-grade Physical Education students. Journal of teaching in Physical Education, 24(1), 88-102.

Bay-Hinitz, A. K., Peterson, R. F. \& Quilitch, H. R. (1994). Cooperative games: a way to modify aggressive and cooperative behaviors in young children. Journal of applied behavior analysis, 27(3), 435-446.

Bayraktar, G. (2011). The effect of cooperative learning on students' approach to general gymnastics course and academic achievements. Educational research and reviews, $6(1), 62-71$.

Blázquez, B. \& Bofill,A. (2009). Estrategias didácticas para la enseñanza de competencias en Educación Física. En D. Blázquez \& E. Sebastiani (Eds.). Enseñar por competencias en Educación Física (139-162). Barcelona: INDE.

Byra, M. (2006). Reciprocal style of teaching: a positive motivational climate. Comunicación presentada al AIESEPWorld Congress. Jyväskylä (Finlandia), 5 al 8 de julio. Recuperado el 12 de enero de 2012 de http:// www.spectrumofteachingstyles.org/literature

Casey,A.(2010). El aprendizaje cooperativo aplicado a la enseñanza del atletismo en la escuela secundaria. En C. Velázquez (Coord.), Aprendizaje cooperativo en Educación Física. Fundamentosy aplicaciones prácticas (187-199). Barcelona: INDE.

Casey, A. \& Dyson, B. (2009). The implementation of models-based practice in Physical Education through action research. European Physical Education review, 15(2), 175-199.

Casey,A., Dyson, B. \& Campbell, A. (2009).Action research in physical education: focusing beyond myself through cooperative learning. Educational Action Research, 17(3), 407-423.

Cohen, E. G. (1999). Organizzare i gruppi cooperativi. Ruoli, funzioni, attività. Gardolo, TN: Erickson.

Denigri, M., Opazo, C. \& Martínez, G. (2007).Aprendizaje cooperativo y desarrollo del autoconcepto en estudiantes chilenos. Revista de Pedagogía, 28(81), 13-41.

Deutsch, M. (1949).A Theory of Co-operation and competition. Human relations, 2, 129-152.

DeVries, D. L. \& Edwards, K. J. (1972). Student teams and instructional games: their effects on cross-race and cross-sex interaction. (Informen $\left.{ }^{\circ} 137\right)$. Baltimore: John Hopkins University. Recuperado el 13 de junio de 2011 de http:// www.eric.ed.gov. Documento: ED070808.

DeVries, D. L. \& Slavin, R. E.(1976). Teams-Games-Tournament: a final reporton the research. (Informen ${ }^{\circ}$ 217). Baltimore: John Hopkins University. Recuperado el 11 de diciembre de 2010 de http://www.eric.ed.gov. Documento: ED133315.

Díaz Aguado, M. J. \& Baraja, A. (1993). Interacción educativa y desventaja sociocultural. Un modelo de intervención para favorecer la adaptación escolar en contextos inter-étnicos. Madrid: CIDE. 
Dowler, W. (2012). Cooperative Learning and interactions in inclusive secondaryschool physical education classes in Australia. En B. Dyson \& A. Casey (Eds.), Cooperative learning in Physical Education. A research-based approach (150-165). London: Routledge.

Dyson, B. (2001). Cooperativelearning in an elementary Physical Education program. Journal of teaching in Physical Education, 20(3), 264-281.

Dyson, B. (2002). The implementation of cooperative learning in an elementary school physical education program. Journal of teaching in Physical Education, 22(1), 69-85.

Dyson, B., Linehan, N. R. \& Hastie, P. A. (2010). The ecology of cooperative learning in elementary Physical Education classes. Journal of teaching in Physical Education, 29(2), 113-130.

Fernández-Río, J.(2003). El aprendizaje cooperativo en el aula de educación física para la integración en el medio social: análisis comparativo con otros sistemas de enseñanza y aprendizaje. [CD-ROM]. Valladolid: La Peonza.

Garaigordobil, M.(2003). Juegos cooperativos y creativos para grupos de niños de 8 a 10 años. Madrid: Pirámide.

Garaigordobil, M. (2004). Juegos cooperativos y creativos para grupos de niños de 10 a 12 años. Madrid: Pirámide.

Garaigordobil, M. (2005). Juegos cooperativos y creativos para grupos de niños de 6 a 8 años. Madrid: Pirámide.

Garaigordobil, M. (2007). Juegos cooperativos y creativos para grupos de niños de 4 a 6 años. Madrid: Pirámide.

Ghaith, G. M., Shaaban, K. A. \& Harkous, S. A. (2007). An investigation of the relationship between forms of positive interdependence, social support, and selected aspects of classroom climate. System, 35,229-240.

Gillies, R. M. \& Boyle, M. (2010). Teachers' reflections on cooperative learning: Issues of implementation. Teaching and teacher Education, 26, 933-940.

Glover, D. R. \& Midura, D.W.(1992). Team building through physical challenges. Champaign, IL: Human Kinetics.

Goudas, M. \& Magotsiou, E. (2009). Theeffects of a cooperative Physical Education program on students' social skills. Journal of applied sport Psychology, 21(3), 356-364.

Grineski, S. (1989). Effects of cooperative games on the prosocial behavior interactions of young children with and without impairments. [Tesis doctoral]. University of North Dakota. Recuperado el 18 de octubre de 2011 de http:// proquest.umi.com/pqdweb. Documento:AAT 9029602.

Grineski, S. (1996). Cooperative learning in Physical Education. Champaign, IL: Human Kinetics.

Gröben, B. (2005). Kooperatives lernen im spiegel der unterrichtsforschung. Sportpädagogik, 6, 48-52.

Hänze, M. \& Berger, R. (2007). Cooperative learning, motivational effects, and student characteristics: an experimental study comparing cooperative learning and direct instruction in 12th grade physics classes. Learning and instruction, 17(1), 29-41.

Johnson, D. W. \& Johnson, R. T. (1989). Cooperation and competition: Theory and research. Edina, $\mathrm{MN}$ : Interaction Book Company.

Johnson, D. W. \& Johnson, R. T. (1999). Aprender juntos y solos. Aprendizaje cooperativo, competitivo e individualista. Buenos Aires:Aique.

Johnson, D. W. \& Johnson, R. T. (2014). Cooperative Learning in 21st Century. Anales de Psicología, 30(3), 841-851.

Johnson, D. W., Johnson, R. T. \& Holubec, E. J. (1999). Los nuevos círculos de aprendizaje. La cooperación en el aula y la escuela. BuenosAires: Aique.

Johnson, D. W., Johnson, R. T. \& Stanne, M. B. (2000). Cooperative Learning methods: a meta-analysis. Recuperado el 2 de junio de 2011 de www.tablelearning.com/uploads/File/EXHIBIT-B.pdf

Johnson, D. W., Johnson, R. T. \& Taylor, B. (1993). Impact of cooperative and individualistic learning on high-ability students' achievement, self-esteem and social acceptance. Journal of social Psychology, 133(6), 839-844.

Johnson, D. W., Maruyama, G, Johnson, R. T., Nelson, D. \& Skon, L. (1981) Effects of cooperative, competitive, and individualistic goal structures on achievement:A meta-analysis. Psychological Bulletin, 89(1), 47-62.

Kagan, S. (2000). L'apprendimento cooperativo: l'approccio strutturale. Roma: Edizioni Lavoro.

Kirk, T. (1999). Celebrating diversity through cooperative learning and social skills Irisheducational studies, 18(1), 75-90.

Lafont, L. Proeres, M. \& Vallet, C. (2007). Cooperative group learning in a team game: role of verbal exchanges among peers. Social Psychology of education, 10(1), 93-113.

Lavega, P., Planas, A. \& Ruiz, P. (2014). Juegos cooperativos e inclusión en Educación Física. Revista Internacional de Medicina y Ciencias de la Actividad Física y el Deporte, 14(53), 37-51.

López-Pastor, V. M., Barba, J. J., Vacas, R. A. \& Gonzalo, L. A. (2010). La evaluación en educación física y las actividades físicas cooperativas. ¿Somos coherentes? Las posibilidades de la evaluación formativa y compartida. EnC. Velázquez(Coord.), Aprendizaje cooperativo en Educación Física. Fundamentos y aplicaciones prácticas (225-255). Barcelona: INDE.
Marín, M. N. (2007). Efectos de un programa de Educación Física basado en la expresión corporal y el juego cooperativo para la mejora de habilidades sociales, actitudes y valores en alumnado de Educación Primaria. [Tesis doctoral]. Granada: Universidad de Granada.

Marín, S. \& Blázquez, F.(2003).Aprender cooperando: el aprendizaje cooperativo en el aula. Mérida: Dirección General de Ordenación, Renovación y Centros.

Mosston, M. (1978). Enseñanza de la Educación Física. Del comando al descubrimiento. Paidós: Barcelona.

Oortwijn, M. B., Boekaerts, M., Vedder, P. \& Fortuin, J. (2008). The impact of a cooperative learning experience on pupils' popularity, non-cooperativeness and interethnic bias in multiethnic elementary schools. Educational Psychology, 28(2), 211-221.

Orlick, T. (1978). Winning through cooperation.Washington, D.C.: Acropolis.

Orlick, T. (1990). Libres para cooperar, libres para crear. Barcelona: Paidotribo.

Pérez-Pueyo,A.(2005). Estudio del planteamiento actitudinal del área de Educación Física de la Educación Secundaria Obligatoria en la LOGSE. (Una propuesta didáctica centrada en una metodología basada en actitudes). [Tesis doctoral]. León: Universidad de León.

Petersen, R. P., Johnson, D. W. \& Johnson, R. T. (1991). Effects of cooperative learning on perceived status of male and female pupils. The journal of social Psychology, 131(5), 717-735.

Piercy, M., Wilton, K. \& Townsend, M. (2002). Promoting the social acceptance of young children with moderate-severe intellectual disabilities using cooperativelearning techniques. American journal on mental retardation, 107(5), 352360.

Polvi, S. \& Telama, R. (2000). The use of cooperative learning as a social enhancer in Physical Education. Scandinavian journal of educational research, 44(1), 105-115.

Prieto, J.A. \& Nistal, P. (2009). Influencia del aprendizaje cooperativo en educación física. Revista Iberoamericana de Educación, 49(4), 1-8.

Provost, P. \& Villeneuve, J. (1980). Jouons ensemble. Jeux et sports coopératifs. Montreal: Les editions del'homme.

Pujolás, P. (2008). El aprendizaje cooperativo. 9 ideas clave. Barcelona: Graó.

Slavin, R. E. (1999). Aprendizaje cooperativo. Teoría, investigación y práctica. Buenos Aires:Aique.

Stanne, M. B., Johnson, D. W. \& Johnson, R. T. (1999). Does competition enhance or inhibit motor performance: a meta-analysis. Psychological Bulletin, 125(1), 133-154.

Strachan, K. (1996). Cooperative learning in a secondary school Physical Education program. [Tesis demáster]. McGill University, Montreal. Recuperado el 22 de diciembre de 2011 de http://proquest.umi.com/pqdweb. Documento: AATMQ29570.

Street, H., Hoppe, D., Kingsbury, D. \& Ma, T. (2004). The Game Factory: using cooperative games to promote pro-social behaviour among children. Australian journal of educational \& developmental Psychology, 4, 97-109.

Tarim, K. \& Akdeniz, F. (2008). The effects of cooperative learning on Turkish elementary students' mathematics achievement and attitude towardsmathematics using TAI and STAD methods. Educational studies in Mathematics, 67(1), 77-91.

Velázquez, C. (2004). Las actividades físicas cooperativas. Una propuesta para la formación de valores a través de la educación física en las escuelas de educación básica. México, D.F.: Secretaría de Educación Pública.

Velázquez, C. (Coord.) (2010). Aprendizaje cooperativo en Educación Física. Fundamentos y aplicaciones prácticas. Barcelona: INDE.

Velázquez, C. (2012). La apedagogía de la cooperación en Educación Física. Laguna de Duero: La Peonza.

Velázquez, C.(2012b). Elaprendizaje cooperativo en Educación Física. La formación de los grupos y su influencia en los resultados. Tándem, 39, 75-84.

Velázquez, C. (2014). Coopedagogía. El enfoque de la pedagogía de la cooperación en Educación Física. En C. Velázquez, J. Roanes \& F. Vaquero(Coord.). Actas del IX Congreso internacional de actividades físicas cooperativas. Vélez Málaga-Torre del Mar, 30 de junio al 3 de julio. [CD-ROM]. Valladolid: La Peonza.

Velázquez, C., Fraile,A. \& López-Pastor, V. M.(2014). Aprendizaje cooperativo en Educación Física. Movimento, 20(1), 239-259.

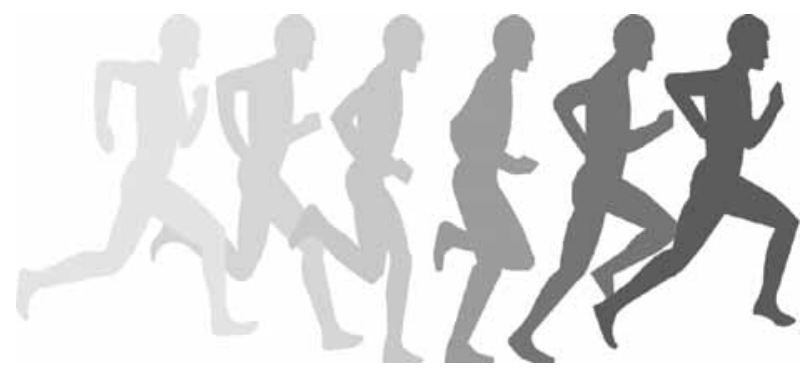

